\title{
Enophthalmos as a sign of metastatic breast carcinoma
}

\author{
Elena Novitskaya PhD, Cornelius Rene MBBS
}

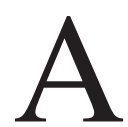
70-year-old woman, who had undergone a right mastectomy 17 years earlier for breast adenocarcinoma, presented with gradual deterioration of vision in her left eye and intermittent diplopia of at least 1 -year duration. Her visual acuity was $20 / 40$ in the left eye and 20/20 in the right. Enophthalmos, ptosis, induration of the periorbital skin, restricted eye movements and incomplete eyelid closure were seen on the left side, along with conjunctival injection with punctate corneal staining, indicative of ocular surface exposure (Figure 1).

Orbital computed tomography (Appendix 1, available at www.cmaj.ca/lookup/suppl/doi:10 .1503/cmaj.120726/-/DC1) showed an ill-defined mass in the left antero-superior orbit. The biopsy results were consistent with estrogen-receptorpositive and HER2-negative metastatic breast carcinoma (Appendix 1). In the absence of metastatic disease elsewhere, the patient was started on letrozole. She may require orbital radiotherapy if her disease progresses.

Enophthalmos has many causes, including orbital fractures, silent sinus syndrome, orbital fat atrophy, orbital varices, orbital inflammation with fibrosis, and orbital tumours. ${ }^{1}$ Orbital metastases are relatively uncommon, accounting for $1 \%-13 \%$ of orbital tumours. ${ }^{2}$ Scirrhous (or fibrosing) breast carcinoma is the most common tumour that metastasizes to the orbit; it causes enophthalmosis by retraction of the orbital contents. ${ }^{2,3}$ A long latency period, averaging 4.56.5 years, usually exists between the initial diagnosis of breast carcinoma and the detection of orbital metastases. ${ }^{2}$

A careful history and examination, assisted by orbital imaging (computed tomography or magnetic resonance imaging), are essential for diagnosing orbital metastases. ${ }^{4}$ Enophthalmos may indicate the presence of metastatic breast cancer and may occur even if the primary lesion is occult. ${ }^{5}$ Histopathologic examination should be considered, even for patients with a known history of systemic cancer, before planning treatment. ${ }^{4}$
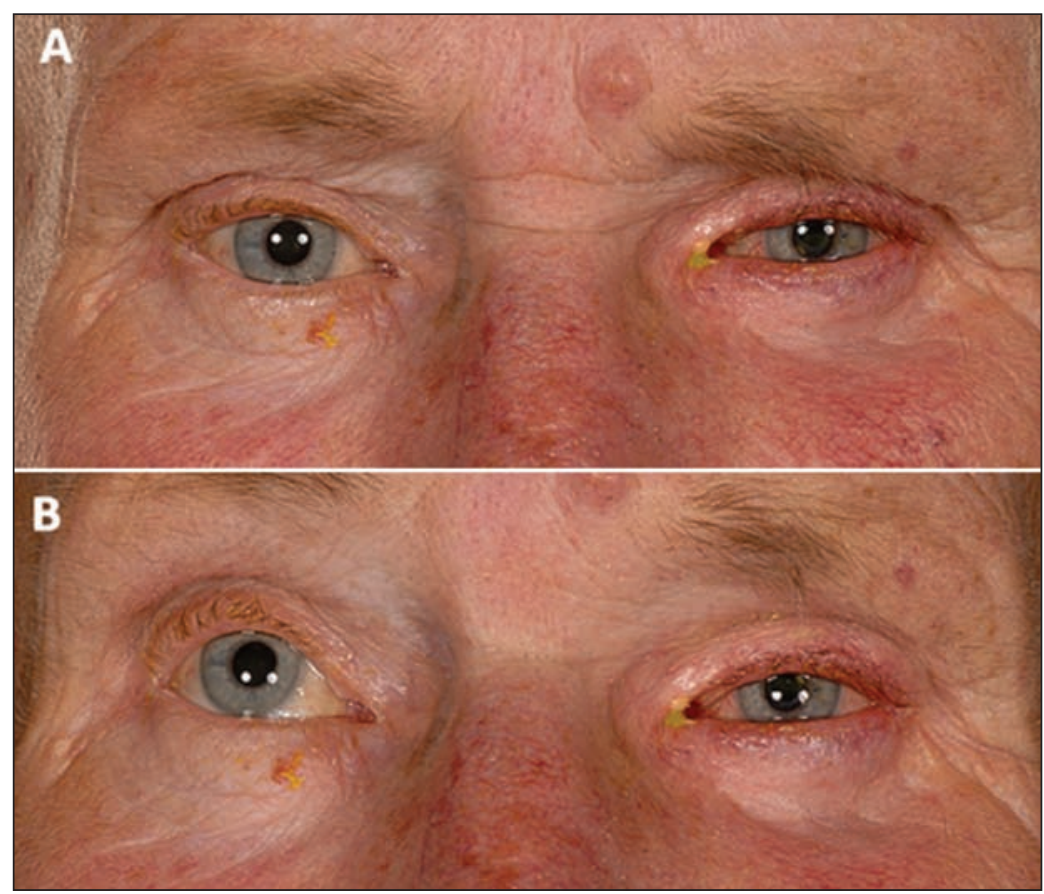

Figure 1: A 70-year-old woman with metastatic breast carcinoma and enophthalmos, ptosis, thickening of the eyelids and conjunctival injection on the left side (A). On attempted upgaze (B), her left eye fails to elevate.

\section{References}

1. Hamedani M, Pournaras JA, Goldblum D. Diagnosis and management of enophthalmos. Surv Ophthalmol 2007;52:457-73.

2. Ahmad SM, Esmaeli B. Metastatic tumors of the orbit and ocular adnexa. Curr Opin Ophthalmol 2007;18:405-13.

3. Shields JA, Shields CL, Brotman HK, et al. Cancer metastatic to the orbit: the 2000 Robert M. Curts Lecture. Ophthal Plast Reconstr Surg 2001;17:346-54.

4. Char DH, Miller T, Kroll S. Orbital metastases: diagnosis and course. Br J Ophthalmol 1997;81:386-90.

5. Shields CL, Stopyra GA, Marr BP, et al. Enophthalmos as initial manifestation of occult, mammogram-negative carcinoma of the breast. Ophthalmic Surg Lasers Imaging 2004;35:56-7.

Clinical images are chosen because they are particularly intriguing, classic or dramatic. Submissions of clear, appropriately labelled high-resolution images must be accompanied by a figure caption and the patient's written consent for publication. A brief explanation (250 words maximum) of the educational significance of the images with minimal references is required.
Competing interests: None declared.

This article has been peer reviewed.

\section{Affiliations:}

Ophthalmology

Department, Addenbrooke's Hospital, Cambridge, UK

Acknowledgement: The authors thank Dr. Andrew Dean for his help in preparing the histopathology slides.

Correspondence to: Elena Novitskaya, elena.novitskaya@gmail.com

CMAJ 2013. DOI:10.1503 /cmaj.120726 